\title{
Técnicas de mejora en la calidad asistencial en hemodiálisis
}

\author{
Virginia A. Marín Fábrega, Ma José Marqués Racionero, Macarena Peña Ortega, Mª Luis Molina Conde
}

\section{Club de Hemodiálisis Nefrolinares. Linares. Jaén}

\section{Introducción:}

En el cuidado integral ofrecido al paciente en programa de hemodiálisis (HD) es fundamental conseguir la menor variabilidad posible con la mayor calidad del proceso asistencial. Para lograrlo existen diversas técnicas que anteriormente pertenecían a otros campos y que se han adaptado para su aplicación en sanidad y en concreto en el de la HD.

\section{Objetivos:}

Analizar las técnicas que minimizan la variabilidad asistencial en HD.

\section{Material y método:}

Estudio de revisión bibliográfica.

DESARROLLO FEEDBACK: Información periódica y sistemática de los resultados personales, con el fin de mejorar tanto los individuales como los del resto del equipo. Es útil tanto en para el paciente como para el profesional.

BENCHMARKING: Análisis de los resultados propios y comparación con los obtenidos por otras unidades, consideradas como referentes de calidad, para hacer una estimación de los beneficios que obtendría nuestra unidad si trabajase de la misma forma. No es una actividad aislada sino un proceso de aplicación de un conjunto de acciones en un orden particular con una secuencia periódica sistemática y repetida de forma organizada.
PDCA: Consecución de cuatro pasos de forma ordenada.

Paso 1. Planificar (Plan). Analizar el proceso para determinar qué cambios necesita y de qué manera.

Paso 2. Hacer (Do). Realizar los procedimientos decididos en la fase anterior.

Paso 3. Chequear (Check). Verificar lo implantado para conocer los resultados obtenidos y compararlos con los esperados.

Paso 4. Actuar (Action). Estudiar los resultados obtenidos y tomarlos como punto de partida de un nuevo ciclo.

AMFE: Análisis modal de fallos y efectos es una técnica de detección de errores presentes y de prevención de errores futuros que ayuda a relacionar cada fallo detectado con sus consecuencias. Analiza cada fallo según su Detectabilidad, Frecuencia, Gravedad e Índice de Prioridad de riesgo.

MANUAL DE ESTANDARES DE HD: Modelo basado en patrones de referencia. Garantiza que la unidad que lo implante estará centrada en alcanzar la mejor calidad en sus funciones. Ayuda a cumplir las expectativas de los profesionales, pacientes y familiares. Mejora las competencias del personal sanitario para obtener los mejores resultados. Utiliza para su elaboración técnicas complementarias como el ciclo de Deming.

MAPA DE RIESG0: Representación gráfica de los riesgos, tanto posibles como reales, que se detec- 
tan en la unidad de HD. Cada unidad presentará un mapa de riesgo único y adaptado a su problemática individual e irá cambiando a lo largo del tiempo. Usa indicadores de forma sistematizada e informaciones descriptivas sobre cada unidad. Integra el uso de técnicas como el AMFE.

\section{Conclusiones:}

Los profesionales sanitarios que estamos en contacto con los pacientes en programa de HD tenemos a nuestro alcance herramientas suficientes para conseguir la mejora de la calidad asistencial. Las distintas técnicas de mejora continua pueden utilizarse de forma individual o complementaria. La aplicación sistematizada de los procesos de calidad asistencial aumenta la satisfacción tanto del personal como de los pacientes y favorece la mejora de la atención integral del paciente en programa de HD y sus resultados.

Palabras Clave: Calidad asistencial, Mapa de riesgo, Seguridad, Hemodiálisis.

\section{Referencias Bibliográficas}

1. Manual de Estándares de Unidades de Hemodiálisis. Agencia de Calidad Sanitaria de Andalucía. Consejería de Salud. Mayo 2012. ISBN: 978-8415318-73-6.

2. Arenas MD et al. Aseguramiento de la calidad y certificación de una unidad de HD según normas IS0 9000. Nefrología 2003;23:37-46.

3. El análisis modal de fallos y efectos (AMFE). Una herramienta muy útil para la seguridad del paciente. Pedro Ruiz López y Carmen González Rodríguez-Salinas. Jano 27 de junio-3 julio 2008. No 1702.

4. Aranz JM, Moya, C. Seguridad del paciente y calidad asistencial. Rev. Calid. Asist. 2011; 26 (6): 331-2. 